\title{
Frontières
}

\section{Éducation de la relation à la mort}

\section{Josef Brynczka}

Volume 13, numéro 1, automne 2000

\section{La mort au tableau noir}

URI : https://id.erudit.org/iderudit/1074243ar

DOI : https://doi.org/10.7202/1074243ar

Aller au sommaire du numéro

\section{Éditeur(s)}

Université du Québec à Montréal

ISSN

1180-3479 (imprimé)

1916-0976 (numérique)

Découvrir la revue

Citer cette note

Brynczka, J. (2000). Éducation de la relation à la mort. Frontières, 13(1), 29-32. https://doi.org/10.7202/1074243ar

\section{Résumé de l'article}

Faut-il éduquer les enfants à la mort ? L'article qui suit suggère de poser la question directement aux intéressés. Mais si on considère que l'évolution du concept de la mort, surtout dans sa dimension existentielle, passe par la relation à la naturalisation de la mort, à l'ambivalence corporelle et à l'ambivalence relationnelle, avant que l'on puisse aborder de front la relation à la mort en soi, la question mérite alors d'être reformulée : faut-il éduquer les enfants à la relation à la mort ?

Les activités éducatives proposées s'inspirent de quatre (4) thèmes liés aux relations à la mort : naturaliser la mort, accepter le corps mortel, apprendre à vivre les pertes relationnelles, admettre la mort dans la vie. Le sondage effectué auprès des élèves montre des réactions très favorables à l'introduction de ces thèmes dans leur cadre éducatif. Cela devrait questionner l'attitude tabouisante de l'adulte.
Ce document est protégé par la loi sur le droit d'auteur. L'utilisation des services d'Érudit (y compris la reproduction) est assujettie à sa politique d'utilisation que vous pouvez consulter en ligne.

https://apropos.erudit.org/fr/usagers/politique-dutilisation/ 


\section{Résumé}

Faut-il éduquer les enfants à la mort? L'article qui suit suggère de poser la question directement aux intéressés. Mais si on considère que l'évolution du concept de la mort, surtout dans sa dimension existentielle, passe par la relation à la naturalisation de la mort, à l'ambivalence corporelle et à l'ambivalence relationnelle, avant que l'on puisse aborder de front la relation à la mort en soi, la question mérite alors d'être reformulée : faut-il éduquer les enfants à la relation à la mort?

Les activités éducatives proposées s'inspirent de quatre (4) thèmes liés aux relations à la mort : naturaliser la mort, accepter le corps mortel, apprendre à vivre les pertes relationnelles, admettre la mort dans la vie. Le sondage effectué auprès des élèves montre des réactions très favorables à l'introduction de ces thèmes dans leur cadre éducatif. Cela devrait questionner l'attitude tabouisante de l'adulte.

Mots clés : mort - ambivalence - pédagogie de la mort

\section{Abstract}

Should we educate children about death? The following article suggests asking the children directly. In considering that the evolution of the concept of death, specially in its existential dimension, goes through the normalization of death, through the relation to corporal ambivalence and through the relation to relational ambivalence before tackling directly the relation to death in itself. The question begs to be asked slightly differently: should we educate children about their relation towards death.

The proposed educational activities are rooted in four themes of relation towards death: normalizing death, accepting the mortal body, learning to live with relationship loss and admitting the presence of death in life.

The survey among children shows very positive responses to the presence of those themes in their educational environment. That should raise questions with the taboo perpetuating attitude of adults.

Key words : death-ambivalence - death pedagogy

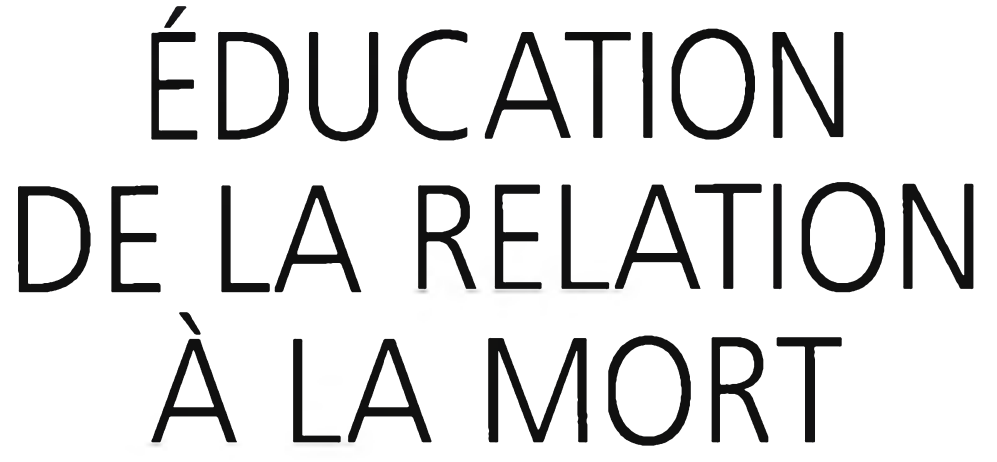

“ NOUS DEVONS APPRENDRE À MOURIR POUR VIVRE. "

- NIETZSCHE

Josef Brynczka,

professeur au Département des sciences de l'éducation et directeur du Centre d'études sur la mort de I'UQAM.

L'éducation à la mort ne jouit pas d'une considération positive dans notre société. Faut-il éduquer à la mort ? À quoi éduquer si la mort est insaisissable ? La mort n'est-elle pas un mystère et n'est-il pas préférable de la traiter comme telle, intrigante et inaccessible ? Ces questions, et beaucoup d'autres, semblent mettre en doute la pertinence de l'éducation à la mort. Les aspects désagréables, ou carrément " morbides " attribués à cet événement de notre existence contribuent à la reléguer aux oubliettes. Pourtant, nous continuons de croire qu'une éducation à la mort demeure une voie privilégiée pour éveiller les enfants à son incontournable réalité1.

Devant l'évidence de sa présence, chaque société s'exprime par des représentations symboliques et des concepts qui donnent alors l'illusion de la saisir. Il existe des recherches traitant plus particulièrement des dimensions cognitives de la mort. On désire alors savoir, notamment, comment les enfants construisent le concept de mort en tenant compte de l'irréversibilité de la vie, de la temporalité, de la finitude et de la réciprocité. D'autres recherches portent sur la dimension existentielle de la mort. La dimension existentielle " repose sur l'expérience personnelle subjective ${ }^{2}$. Cette dimension considère le fait que nous cheminons seuls dans l'existence avec le sentiment de notre mortalité, que nous avons conscience d'une telle existence et que nous serons seuls à vivre notre mort.

\section{LES DIMENSIONS EXISTENTIELLES ET COGNITIVES DE LA MORT}

Examinons d'abord de plus près la dimension existentielle. Il est certes une évidence de souligner que nous ne pouvons pas nous targuer d'avoir une expérience directe de notre mort. Par contre, un certain nombre d'expériences existentielles - le décès d'une autre personne, une séparation, la solitude, la maladie, le sentiment de notre contingence, la transformation du statut identitaire, etc. - nous mettent en relation avec elle. En fait, le rapport à la mort ressort d'événements forts et marquants pour un individu, événements qui lui rappellent sa fragilité de mortel. À titre d'exemple, une personne atteinte du sida ressent sa vie à travers cette possibilité ouverte de la mort. C'est une expérience existentielle extrême que nous préférons éviter et que nous ne souhaitons à personne mais nous sommes exposés à sa présence. L'éveil à la fragilité de la vie, qui initie l'être humain au sentiment de sa mortalité, pourra survenir lors d'une 
expérience beaucoup moins forte et malheureuse. Pour les enfants, cet éveil se réalise lors d'une maladie, après la lecture d'un conte ou lors de la mort d'un animal. Il va de soi que la discussion avec un parent ou un enseignant devient un atout inévitable pour aider l'enfant à réaliser le sens de ce qu'il vit et de ce qu'il ressent lors d'une telle expérience existentielle. En somme, la dimension existentielle renvoie à la fragilité mortelle de la vie. Elle est donc le vécu de l'opposition que représente le tandem viemort et, en même temps, elle est le vécu de la complémentarité entre ces deux inséparables composantes de l'existence; elle est aussi cette capacité qui permet d'expériencier ce genre d'existence, l'existence en rapport à la mort.

$\mathrm{Au}$ niveau cognitif, la vie et la mort sont mutuellement nécessaires pour se définir l'une et l'autre. Par exemple, la vie humaine est définie par sa durée : elle commence après la fécondation et se termine à la mort. Aussi, on définit la vie par sa capacité de se reproduire. La reproduction est essentielle pour continuer la vie parce que la mort survient, ou parce qu'un être vivant n'est pas immortel. On ne peut discuter de la vie sans faire intervenir le concept de mort, ni, en revanche, discuter de la mort sans le concept de vie. Nous sommes alors en présence de ce que nous désignerons par le terme d'" ambivalence ». Dans son sens premier, l'ambivalence signifie ce qui comporte deux composantes de sens contraire. On parlera d'ambivalence pour signaler une expérience existentielle lors de laquelle une personne s'éveille au sentiment que la mort et la vie sont complémentaires, mais incompatibles. Cette notion d'ambivalence va nous permettre de mieux comprendre comment les enfants construisent le concept de mort. À cet égard, l'éducation à la mort que nous proposons s'appuie sur ce concept d'ambivalence qui prend en compte à la fois les dimensions existentielle et cognitive.

Alors que la dimension existentielle relève de l'expérience vécue par chacun dans son intimité, la dimension cognitive examine comment un enfant construit le concept de mort. En pratique, la dimension cognitive s'intéresse aux relations que fait un enfant entre une situation vécue lors de laquelle il expérimente un aspect de la mort, et des éléments conceptuels relatifs à la mort. Il s'agit pour l'enfant de mettre en relation, notamment, le temps de la vie et la mort. Nous travaillons avec l'hypothèse selon laquelle le concept de la mort fait référence à cinq aspects : dynamique (attachement/détachement), changement, irréversibilité biologique, irréversibilité relationnelle (universalité) et aspect métaphysique (sens). Chacun de ces aspects renvoie à un stade du développement de l'enfant, comme nous le signalerons ci-après.

\section{LES ÉTAPES DE L'AMBIVALENCE}

La relation à une expérience d'ambivalence peut être passive ou active. Dans la relation passive, la mort est subie ; dans la relation active elle reste une tâche à réaliser. Reléguer cette tâche à la fin de la vie serait choisir la relation passive tout au long de la vie. Par contre, considérer la relation à la mort comme une tâche à réaliser suppose un travail sur cette relation. Dans cet esprit, nous avons proposé à vingt-huit élèves de 8 à 12 ans, regroupés en deux classes multiâges, une démarche d' "éducation à la mort pour la vie ". Nous avons par la suite recueilli leur opinion et leurs réactions sur cette démarche. Enfin, nous avons cherché à savoir quelle importance les enfants accordaient au thème de la mort à l'école.

L'éducation qui considère l'ambivalence de l'aspect existentiel de la mort s'intéresse à la manière de vivre, à chaque âge de la vie, ce qui rend la vie et la mort complémentaires et incompatibles. Pour chaque étape de la vie, chaque enfant adopte une postule singu- cation à la mort. L'ambivalence, ici, prend le sens de cette dynamique perpétuelle de l'attachement et du détachement qui constitue un enjeu éducatif important de 0 à 12 ans.

Chez les enfants de 3 à 5 ans, on constate l'expression de la dimension " changement " qui caractérise le concept de la mort ${ }^{4}$ : le mort s'endort, il peut donc se réveiller, mais pour vivre ailleurs. Dans cet " ailleurs " se trouve déjà impliquée une séparation d'avec un proche, mais non d'avec la vie. La mort est ici vécue comme un changement de vie. Comme pour beaucoup d'autres changements, on n'a pas à le juger, il est seulement permis de le constater. Cette compréhension de la mort inspire, en éducation, le principe de "naturalisation " de la mort. C'est notre premier principe. Cette dimension de changement signale cette nouvelle ambivalence (changement stable) qui renvoie au cycle $\mathrm{vie} / \mathrm{mort} /$ renaissance.

Pour l'enfant de $\mathbf{6}$ à $\mathbf{8}$ ans, la mort est caractérisée par l'irréversibilité biologique. Le mort ne respire pas, n'entend pas, ne bouge pas. Cette perte des capacités sensorielles est permanente. Aussi la relation à la mort est vécue à travers l'expérience de la déchéance corporelle. L'enfant se rend compte que ce corps est en croissance, que sa puissance augmente et qu'il est même doté d'une capacité de procréation tout en étant appelé à

\section{SCHÉMA DE L'AMBIVALENCE CORPORELLE AMBIVALENCE DU CORPS}

corps appelé à

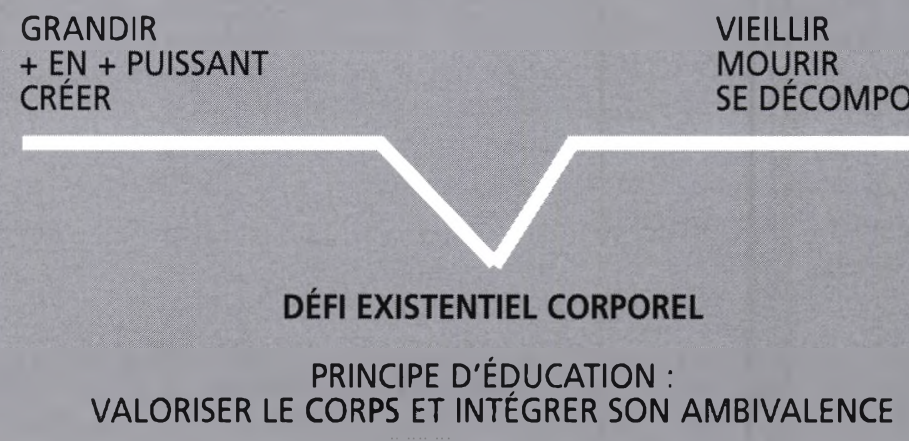

lière. Voyons les étapes du développement en fonction de l'expérience d'ambivalence.

De 0 à 2 ans, l'enfant vit l'aspect dynamique du concept de la mort qui se manifeste par des expériences d'attachement et de détachement ${ }^{3}$. Elle accompagnera toujours la relation à la mort. Il faudra alors en tenir compte dans l'édu- vieillir, à mourir et même à se décomposer : "Quand on a 7 ans ou 77 ans, penser d'avoir un corps mortel qui se décompose, il y a de quoi faire réagir tout être humain ${ }^{5}$ ". L'enfant doit se situer par rapport à cette relation ambivalente à son corps : il l'accepte et l'intègre à son vécu ou il le fuit et l'enterre sous le silence. Il vit alors, par le même 
fait, une expérience ambivalente de la mort. Réussir cette relation, c'est relever le " défi existentiel corporel ", donc apprendre à accueillir l'ambiguïté corpo- sans cesser d'aimer ». C'est là notre troisième principe d'éducation à la mort.

La présentation ci-jointe illustre cette ambivalence et le défi.

\section{AMBIVALENCE DES LIENS}

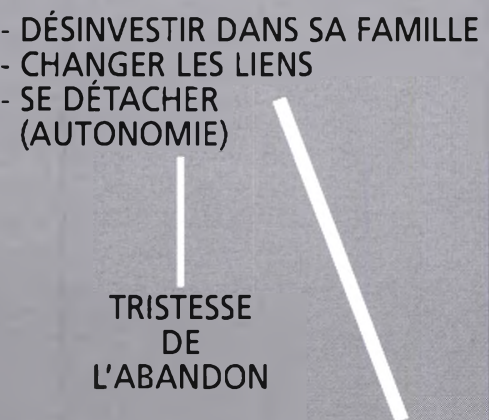

DÉFI EXISTENTIEL RELATIONNEL

PRINCIPE D'ÉDUCATION : SE DÉTACHER SANS CESSER D'AIMER
- INVESTIR AUPRÈS DES PAIRS

- CRÉER DES NOUVEAUX LIENS

- RISQUER DE NOUVEAUX

ATTACHEMENTS

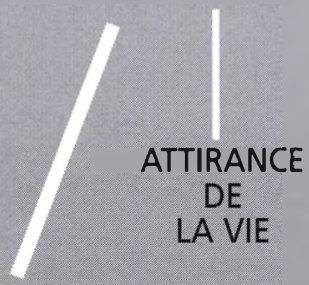

dans le livre choisi pour cet atelier, d'un dialogue entre une grand-mère et un petit-fils où il est interdit d'utiliser les mots «oui » et "non ». À la fin du livre, le petit-fils interroge sa grandmère sur sa mort. Cette lecture est suivie d'une discussion. L'animatrice accueille les questions et les réflexions des élèves.

Les autres ateliers se déroulaient, mutatis mutandis, de manière analogue.

\section{NATURALISATION DE LA MORT}

\section{- Activités}

- les changements dans la nature,

- les dichotomies (jour/nuit; sommeil/réveil....vie/mort),

- dessin : qu'est-ce que la vie pour toi ?

- lecture : Ni oui ni non ${ }^{7}$,

- échange sur le texte, sur la possibilité de la mort de la grand-mère.

\section{L'AMBIVALENCE DU CORPS}

relle, et par cela réaliser sa relation à la mort-fin. S'éduquer à la souffrance et à la déchéance du corps équivaut à " dédramatiser la mort inscrite dans le corps ». Cette dernière idée devient notre deuxième principe d'éducation à la mort.

De 9 à 12 ans, la mort est perçue comme une cessation du processus de vie. La mort occasionne la fin de la vie, non pas en tant que facteur extérieur, mais comme un processus propre à la nature humaine. Elle est irréversible et irrévocable. La mort fait entrer l'être humain dans la finitude. Cependant, dans la pratique, elle est vécue à travers la dynamique sociale propre à cette période de l'enfance, liée au détachement de la famille et à l'établissement de nouveaux liens avec les pairs.

L'enfant se trouve devant un dilemme qui le plonge dans une nouvelle ambivalence. Il doit, d'une certaine manière, se détacher de sa famille, se désinvestir et risquer de perdre certains liens avec ses parents. En même temps, il doit investir dans des relations avec ses pairs ; il doit créer de nouveaux liens et risquer des attachements. L'expérience du détachement d'avec ses parents apprend à l'enfant que chaque attachement contient en lui-même la possibilité d'une rupture. Il est alors placé devant un " défi existentiel relationnel ": comment vivre avec la constatation que chaque lien contient déjà une rupture. La relation à ce défi reflète la relation concrète à la mort. Relever le défi existentiel relationnel conduit à apprendre à "se détacher
L'adolescence réfère au défi existentiel métaphysique ${ }^{6}$, c'est-à-dire à une autre sorte de relation à la mort, vécue, cette fois-ci, comme une ambivalence qui résulte de la contradiction entre le désir de l'absolu et la constatation de sa propre contingence. Cette ambivalence est propre à l'adolescence. Étant donné l'âge de notre clientèle, ce défi ne concerne pas les sujets qui ont participé aux activités.

\section{NOS ATELIERS}

Les principes d'éducation mentionnés ci-haut ont inspiré l'élaboration de quatre ateliers. Chaque atelier est composé de différentes activités réparties sur une journée. Les activités d'éducation à la mort étaient insérées aux activités habituelles de la classe : elles étaient animées par une assistante de recherche en présence de l'enseignante. Les aspects liés à la mort étaient toujours entremêlés avec les aspects liés à la vie.

À titre d'exemple, l'atelier 1 avait pour objectifs de : 1) familiariser les élèves avec des changements dans la nature ; 2) d'identifier des dichotomies de la vie ; 3) de constater le naturel de ces changements, y compris le cycle mort/vie ; 4) d'apprécier la vie malgré la présence de l'événement de la mort. La pédagogie intégrée utilisée permettait de saisir avec une grande spontanéité les opportunités d'intervention afin de réaliser ces objectifs. La seule planification effectuée d'avance portait sur le choix d'une lecture dans le cadre $\mathrm{du}$ cours de français. Il est question,
- Objectifs: 1) identifier des qualités et des faiblesses dans mon corps ; 2) sensibiliser à accueillir les limites et le potentiel du corps (préparer à l'intégration de l'ambivalence corporelle)

- Activités

- observer les changements dans mon corps,

- trouver les cinq qualités de mon corps que j'aime le plus,

- raconter l'expérience d'une maladie et de la guérison,

- trouver une caractéristique de mon corps que je n'aime pas,

- accueillir dans mon corps une qualité appréciée et une qualité que je n'aime pas en utilisant la visualisation $^{8}$.

\section{VIVRE UNE SÉPARATION}

- Objectifs : 1) identifier les émotions liées à la séparation ; 2) identifier des forces pour intégrer les émotions ; 3) sensibiliser à la relation aux pertes (ambivalence relationnelle),

\section{Activités :}

- lecture : Ma tante Marie Blanche ${ }^{9}$, - échange : séparations, émotions, comportements, forces,

- jeu : ma peur et mon "magicien » pour la dépasser,

- dessin : dessine une situation où tu as vécu une perte ou une séparation, - échange sur les dessins et comment cette situation pourrait être vécue si on lui appliquait la force intérieure que chacun a identifiée, - expériences de donner et de recevoir. 
4. LA MORT FAIT APPRÉCIER LA VIE

- Objectifs : 1) identifier la relation à

l'événement de la mort ; 2) identifier les notions de mort et de vie ; 3 ) prendre conscience de la présence de la mort dans la vie ; 4) sensibiliser au rapport entre la mort et la vie,

- Activités

- dessin : qu'est-ce que la mort pour toi ?

- échange sur les dessins,

- lecture : L'horloge s'est arrêtée ${ }^{10}$

- échange : les circonstances de la mort, l'enterrement, l'image de la chenille et du papillon, les émotions, l'importance des souvenirs, s'ouvrir à la vie,

- dessin : qu'est-ce que la vie pour toi ?

- échange sur les dessins et sur la valeur de la vie.

\section{SONDAGE}

Le sondage a été effectué auprès des vingt-huit élèves ayant participé aux ateliers. Les élèves étaient regroupés en deux classes multi-âges dans une école alternative du Québec. L'âge des élèves variait de 8 à 12 ans. Ils ont répondu aux questions suivantes :

\section{QU'AVEZ-VOUS APPRIS?}

Trois enfants de 8 ans et deux de 10 ans ont répondu : "Rien ". Les autres ont constaté : avoir moins peur, mieux connaître la vie et la mort, parler de ce qui est difficile à dire, comprendre la séparation, connaître l'opinion des autres.

\section{LAQUELLE DES ACTIVITÉS AVEZ-}

\section{VOUS LE PLUS AIMÉE ?}

L'activité sur la mort (atelier $\mathrm{n}^{\circ} 4$, première partie) a été choisie par quinze élèves et celle sur la vie (deuxième partie de l'atelier $n^{\circ} 4$ ) par six élèves, les autres activités choisies étaient : séparation, peur et magicien, changements... etc. L'explication de leur choix tourne autour de : "Parce que j'avais un peu peur, maintenant je suis plus rassuré ".

\section{QUELLE ACTIVITÉ AVEZ-VOUS LE MOINS AIMÉE ?}

La séparation obtient le plus grand nombre de choix : onze élèves. Neuf élèves ont aimé toutes les activités. Les élèves qui n'ont pas aimé " la séparation " expliquent : "Cela pourrait m'arriver ".

\section{EST-IL IMPORTANT POUR TOI DE PAR-} LER DE LA MORT ?

Seulement trois enfants ont répondu " non ". Parmi les vingt-cinq élèves qui ont répondu " oui ", plusieurs ont souli- gné leur réponse. Les réponses négatives semblent être liées, dans deux cas, à l'idée de passage dans la lumière et, dans un cas, à celle de mauvais souvenirs.

\section{RÉSULTATS ET CONCLUSIONS}

La très grande majorité des enfants a apprécié tous les ateliers. Les apprentissages qu'ils ont réalisés ont touché les émotions et les connaissances. Le thème vedette se révèle être celui de la mort. Il est probable que ce soit la présence inattendue de cette problématique à l'école qui a suscité cet intérêt. Il semble que la démarche progressive, qui commence par les changements naturels avant d'aborder les changements et les pertes personnelles, prépare le terrain pour le thème de la mort. De plus, aborder ce sujet leur permet "d'avoir moins peur ", d'apprendre à en parler et de partager ce vécu difficile avec les autres.

Le thème qui a provoqué le plus de réactions d'appréhension chez les élèves est celui de la séparation. Il est probable que ces réactions soient liées à la période dans laquelle se trouvent nos sujets ; ils se situent dans la période du défi relationnel. Ce thème serait pour eux une vraie et concrète expression de la mort. De plus, cet événement - selon leurs dires et à ce moment de leur vie - serait le plus menaçant.

La constatation la plus importante pour nous est la confirmation par ces élèves de l'importance de parler de la mort à l'école. Considérant les refus que nous avons essuyés avant d'obtenir l'accès à cette école afin d'expérimenter notre démarche d'éducation à la mort, il faut se rendre à l'évidence que c'est un problème d'adulte et non pas celui de l'élève. Au nom de la protection de l'enfant, on l'empêche d'approfondir la relation avec son existence, comme si on oubliait que l'occultation de la mort escamote la vie : " [...] C'est celui qui n'accepte pas la vie qui n'accepte pas la mort, celui qui ne peut entendre ce qu'il en est de la vie, qui ne peut accepter d'entendre ce qui en est de la mort [...] ${ }^{11} »$.

Dans la même ligne de pensée que cette démarche d'éducation à la mort, deux autres recherches ${ }^{12}$ ont confirmé nos constatations, à savoir l'intérêt des élèves de 9-12 ans pour le thème de la mort et l'importance de l'aborder à l'école.

Les jeunes sont intéressés par l'éducation à la mort et même, ils la réclament, surtout quand elle donne une signification à la vie. À leur instar, quand les adultes seront-ils aussi ouverts?

\section{Notes}

1 La présente recherche a fait l'objet d'une communication au 5e Congrès des Sciences de l'éducation de langue française (AFDEC), qui s'est tenu du 18 au 20 août 1997 à l'Université de Moncton.

2 A. MASLOW, Vers une psychologie de l'être, Paris, Fayard, 1972, p. 12

3 J. BOWLBY, La perte, tristesse et dépression, Paris, PUF, 1984

4 R. LONETTO, Dis, c'est quoi quand on est mort?, Paris, Esthel, 1988, p. 43

5 J-P. GÉLINAS, "Quand la mort empiète sur le jour ", Santé mentale au Québec, vol. 7, no 2, 1982 .

6 J. BRYNCZKA, "Quelques pistes d'éducation par la mort... pour la vie ", Frontières, vol. 6, no 1, printemps 1993.

7 Les livres pour enfants sont choisis en fonction des thèmes à aborder dans chaque atelier ; ils sont lus aux enfants et constituent des déclencheurs de discussions.

M. DUFRESNE, Ni oui ni non, Paris, Hachette, 1989

8 J. CLÉMENT, " Le balai magique ", Relaxation et visualisation par l'imagerie mentale, Sherbrooke, Éditions du IIIe millénaire, 1991

9 R. SOULIÈRES, $M a$ tante Marie Blanche, Montréal, Québec/Amérique, 1980

$10 \mathrm{~J}$. DUBÉ, L'horloge s'est arrêtée, Montréal, P. Tisseyre, 1990.

11 G. RAIMBAULT, "La mort et l'enfant», dans Fin de vie sous la direction de C.-H. RAPIN, Lausanne, Payot, 1989, p. 112

12 P. FAILLETAZ, Faut-il parler de la mort aux enfants et comment ?, thèse de maîtrise, Faculté de psychologie, Université de Genève, 1996 ; S. LAPOINTE, Atelier d'éducation à la mort, document inédit, produit dans le cadre du cours EDU 7215, L'enfant et la mort, Université du Québec à Montréal, 1997. 